\title{
Trophic downgrading decreases species asynchrony and community stability regardless of climate warming
}

\author{
Felipe Rezende ${ }^{1}$, Pablo Antiqueira ${ }^{2}$, Owen Petchey ${ }^{3}$, Luiz Velho ${ }^{4}$, Luzia Rodrigues ${ }^{5}$, and \\ Gustavo Romero ${ }^{2}$ \\ ${ }^{1}$ Universidade Estadual de Campinas \\ ${ }^{2}$ State University of Campinas (UNICAMP) \\ ${ }^{3}$ University of Zurich \\ ${ }^{4}$ Universidade Estadual de Maringá (UEM) \\ ${ }^{5}$ NUPELIA
}

March 22, 2021

\begin{abstract}
Theory and some evidence suggest that biodiversity promotes stability. However, evidence of how trophic interactions and environmental changes modulate this relationship in multitrophic communities is lacking. Given the current scenario of biodiversity loss and climate changes, where top predators are disproportionately more affected, filling these knowledge gaps is crucial. We simulated climate warming and top predator loss in natural microcosms to investigate their direct and indirect effects on temporal stability of microbial communites. We also investigated the role of underlying stabilizing mechanisms on community stability. Community stability was insensitive to warming, but indirectly decreased due to top predator loss via increased mesopredator abundance and consequent reduction of species asynchrony and stability. The magnitude of destabilizing effects differed among trophic levels, being disproportionally higher at lower trophic levels (e.g. producers). Our study unravels major patterns and causal mechanisms by which trophic downgrading destabilizes large food webs, regardless of climate warming scenarios.
\end{abstract}

\section{Hosted file}

Rezende et al. - main text.pdf available at https://authorea.com/users/403108/articles/ 514763-trophic-downgrading-decreases-species-asynchrony-and-community-stability-

regardless-of-climate-warming 